Chronic Obstructive Pulmonary Diseases: Journal of the COPD Foundation COOPD

\author{
Original Research
}

\title{
Association of Systemic Endothelial-Derived and Platelet-Derived Microparticles With Clinical Outcomes in Chronic Obstructive Pulmonary Disease
}

\author{
Jorge Lascano, MD ${ }^{1}$ Jason Katz, BS ${ }^{* 1}$ Martin Cearras, MD $^{2}$ Michael Campos, MD $^{3}$
}

\section{Abstract}

Purpose: Endothelial and platelet microparticles (eMPs and pMPs), markers of cellular activation, dysfunction, or apoptosis, have been associated with multiple cardiovascular conditions. Chronic obstructive pulmonary disease (COPD) is associated with cardiovascular comorbidities and platelet/endothelial dysfunction. We analyzed whether eMPs and pMPs are associated with COPD status and/or severity.

Patients and Methods: A total of 58 COPD patients and 19 controls were enrolled and followed for an average of 1.17 years. Characterization of COPD included lung function, Body mass index-airflow ObstructionDyspnea-Exercise (BODE) scores, health-related quality of life, exacerbations, comorbidities, and mortality. Plasma collection to measure eMPs and pMPs via flow cytometry was performed at enrollment as well as during acute exacerbation in 17 participants. We measured pMPs (CD $\left.31^{+}, \mathrm{CD} 41^{+} 31^{+}, \mathrm{CD} 62 \mathrm{P}^{+}\right)$, eMPs (ULEX lectin ${ }^{+}, \mathrm{CD} 51^{+}, \mathrm{CD} 54^{+}, \mathrm{CD} 62 \mathrm{E}^{+}$), the apoptotic $\mathrm{CD} 62 \mathrm{E}^{+} / \mathrm{CD} 31^{+}$ratio, and Annexin V MP.

Results: As a group, COPD participants had no difference in all MP levels studied compared with controls. No significant correlations with diffusion capacity for carbon monoxide, quality of life, and exacerbation status were found in all MPs studied. However, the eMP ULEX and the pMP CD $62 \mathrm{P}^{+}$were higher among COPD Global initiative for chronic Obstructive Lung Disease (GOLD) stage 3 patients compared to controls.

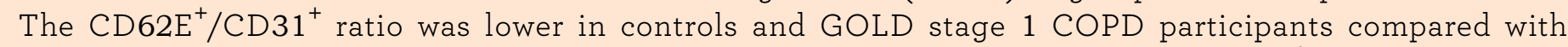
GOLD stage 2/3 COPD participants, suggesting increased apoptosis. eMP ULEX lectin ${ }^{+}$decreased during acute exacerbations and $\mathrm{pMP} 41^{+} 31^{+}$significantly increased as BODE score increased.

Conclusion: After adjusting for comorbidities, most eMPs and pMPs studied do not correlate significantly with COPD status or severity.

\footnotetext{
Abbreviations: endothelial microparticles, eMPs; platelet microparticles, pMPs; chronic obstructive pulmonary disease, COPD; Body mass index-airflow Obstruction-Dyspnea-Exercise index, BODE; Global initiative for chronic Obstructive Lung Disease, GOLD; microparticles, MPs; platelet endothelial cell adhesion molecule, PECAM; Ulex europeus 1 lection, ULEX; diffusing capacity of the lungs for carbon monoxide, DLCO, 6-minute walk test, 6MWT; health-related quality of life, HRQoL; St George's Respiratory Questionnaire, SGRQ; pulmonary function tests, PFTs; platelet rich plasma, PRP; platelet-poor plasma, PPP; fluorescein isothiocynate, FITC; monoclonal antibody, mAb; Multi-Ethnic Study of Atherosclerosis COPD study, MESA

Funding Support: National Institutes of Health T35 Federal Research Grant

Date of Acceptance: May 26, 2021 | Published Online Date: June 1, 2021

Citation: Lascano J, Katz J, Cearras M, Campos M. Association of systemic endothelial-derived and platelet-derived microparticles with clinical outcomes in chronic obstructive pulmonary disease. Chronic Obstr Pulm Dis. 2021;8(3):382-395. doi: https://doi.org/10.15326/ jcopdf.2021.0211
}

1 Pulmonary and Critical Care Division, Department of Internal Medicine, UF Health-Shands Hospital, University of Florida, Gainesville, Florida, United States

2 Advent Health Medical Group, Central Florida Division, Orlando, Florida, United States
3 Pulmonary and Critical Care Division, Department of Internal Medicine, University of Miami Health Systems, Miami, Florida, United States

* medical student 


\section{Address correspondence to:}

Jason B. Katz

MD-candidate

College of Medicine

University of Florida

Phone : (727)288-8356

Email : jasonbkatz@ufl.edu

\section{Keywords:}

COPD, platelets, lung, endothelium, microparticles

\section{Introduction}

Microparticles (MPs) are 0.1 to $1 \mu \mathrm{m}$ membranebound vesicles present in circulating blood shed from cells during activation, injury, or apoptosis. ${ }^{1,2}$ They encase and transfer molecules such as mRNAs, microRNAs, pro-inflammatory cytokines, and/or proteins and are thought to be important mediators of cell-to-cell communication. ${ }^{2,3}$ MPs contain an externalized phosphatidylserine at their surface and can be differentiated based on the presence of specific transmembrane proteins characteristic of their originating cells. For example, platelet-derived microparticles (pMPs) contain CD31 (platelet endothelial cell adhesion molecule [PECAM]), CD41 (the IIb moiety of glycoprotein IIb/IIIa) and CD62P (P-selectin) and endothelial-derived microparticles (eMPs) contain the Ulex europeus I lectin (ULEX), CD31, CD51 (Integrin a V), CD54 (ICAM-1) or CD62E (E-selectin). ${ }^{4-6} \mathrm{MPs}$ that contain Annexin $\mathrm{V}$ bind to phosphatidylserine and reflect cellular apoptosis regardless of the originating cell. ${ }^{7,8}$

Furthermore, quantitative ratios of certain endothelial microparticles (i.e., CD $62 \mathrm{E}^{+} / \mathrm{CD} 31^{+}$ hereby referenced as apoptotic ratio) have been shown to be a simple, yet robust way to distinguish processes that are activating (i.e., increasing MP shedding, increasing cellular interactions, etc.,) or apoptotic. ${ }^{9,10}$ Specifically, a decreased ratio corresponds to apoptosis while an increased ratio corresponds to activation. The role of each specific MP is still being defined, but in general they are thought to actively participate in endothelial homeostasis and regulation of coagulation activation. $^{11}$

There is growing evidence that circulating MP levels correlate with clinical disease, in particular, prothrombotic states and conditions associated with endothelial dysfunction. Elevations of specific
MPs have been described in atherosclerosis, acute coronary disease, cerebrovascular accidents, uncontrolled arterial hypertension, diabetes, the metabolic syndrome, end-stage renal disease, and pulmonary arterial hypertension. ${ }^{1,12-16}$ In this latter condition, elevations of MP 62E has also been linked to increased mortality. ${ }^{13}$

Chronic obstructive pulmonary disease (COPD) is one condition in which MP level analysis may offer cluesinto its complex, multi-system pathophysiologic mechanism, which has not been fully elucidated. It has been shown that noxious stimuli like cigarette smoke is associated with alveolar epithelial damage and apoptosis. ${ }^{17}$ In addition to the endothelium, platelet dysfunction may play a role in COPD. While recent data suggests that COPD Global initiative for chronic Obstructive Lung Disease (GOLD) ${ }^{18}$ stages 2-4 patients do not have a statistically significant difference in platelet counts compared to age/ gender matched controls, ${ }^{19}$ other studies have shown that increased platelet counts are linked to mortality in this condition. ${ }^{20}$ There is substantial evidence showing COPD patients having many cardiovascular comorbidities, ${ }^{21}$ perhaps offering insight into some studies that have even shown a mortality benefit from anti-platelet therapy. ${ }^{22}$

Based on these observations, in this work we explore the potential role of eMPs and pMPs as prognostic biomarkers in COPD by correlating them with widely used clinical parameters of COPD including GOLD stage, diffusing capacity of the lungs for carbon monoxide, (DLCO), 6-minute walk test (6MWT), health-related quality of life (HRQoL) and lastly Body mass index-airflow ObstructionDyspnea-Exercise (BODE) index. We hypothesized that patients with clinically manifested COPD should have significantly different MP levels compared to controls and that MPs correlate with clinical markers of disease severity.

\section{Methods}

\section{Study Population}

This was a case-control study that also utilized paired comparisons. Prospective participant enrollment occurred on a convenience basis at the Miami Veterans Administration Medical Center. Participants were recruited from pulmonary clinics 
(stable COPD participants), emergency department and inpatient wards (acute exacerbations) and through local advertisements (non-COPD controls). COPD was defined as individuals with a history of smoking (defined as >10 pack years) with a forced expiratory volume in 1 second to forced vital capacity ratio $<0.70$ after administration of a shortacting bronchodilator. ${ }^{23}$ Controls were participants with a history of smoking (either current or former) and preserved lung function. All participants provided written informed consent. The protocol and the informed consent process were reviewed and approved by the University of Miami and the Miami VA institutional review boards. All relevant ethical guidelines set forth by these institutions were strictly followed.

\section{Protocol}

Data collection included a baseline questionnaire (demographics, smoking and occupational history, medications, comorbidities) and a HRQoL via the St George's Respiratory Questionnaire (SGRQ). ${ }^{24}$ For accuracy and to limit recall bias, additional information of medications and comorbid conditions were obtained from review of electronic medical records. All participants, including controls and COPD participants when stable, underwent complete pulmonary function tests (PFTs), including DLCO and a 6MWT. (BODE scores were calculated. COPD patients were considered stable if they were at their baseline state without signs of exacerbation. COPD participants recruited during an acute exacerbation, defined as hospitalization due to worsening of their respiratory symptoms and requiring escalation of respiratory therapy, ${ }^{25}$ were followed at least a month after discharge for reassessment. Exclusion criteria included patients with conditions known to increase independently eMP or pMP levels, such as the presence of an acute coronary syndrome, malignant hypertension, recent cardiopulmonary bypass, heparin-induced thrombocytopenia, antiphospholipid syndrome, thrombotic thrombocytopenic purpura, a transient ischemic attack or new cerebrovascular accident, or diagnosis of multiple sclerosis. In addition, patients with solid organ transplantation, neutropenia, known metastatic or hematologic malignancies, or the inability or unwillingness to perform the $6 \mathrm{MWT}$ or PFTs were excluded. Blood samples to measure MPs were drawn in each patient encounter. Specifically, a single draw for non-COPD controls, a single draw for stable COPD patients, and a single draw for acute exacerbators were completed. Thus, an individual patient received 2 blood draws and subsequent MP level analysis if they belonged in the exacerbator cohort $(n=17)$. For stable patients, samples were obtained before any testing and at rest, to avoid changes that may be due to acute exertion.

\section{Laboratory Methods}

Blood samples were collected from peripheral venipuncture with a butterfly, in two $3.2 \%$ buffered $\mathrm{Na}$ citrate tubes (Becton Dickinson). Samples were kept at room temperature and were processed within 2-3 hours of collection at the Coulter Laboratory at the University of Miami, which specializes in MP measurements. The methods of eMP and pMP assay have been previously thoroughly described. ${ }^{5,6,9}$ In brief, samples from patients and controls were centrifuged at $160 \mathrm{~g}$ for $10 \mathrm{~min}$ to obtain platelet-rich plasma (PRP). The PRP was then centrifuged for 8 minutes at $1000 \mathrm{~g}$ ( 4000 RPM, Shelton microcentrifuge) ${ }^{26}$ to obtain platelet poor plasma (PPP). After this, a 1.0 um filter was used to ensure extraneous proteins and larger vesicles from plasma were removed. Then $50 \mu \mathrm{L}$ of the PPP was incubated with $4 \mu \mathrm{L}$ of each respective monoclonal antibody (mAb) for 20 minutes with gentle shaking using an orbital shaker at $120 \mathrm{rpm}$. The mAbs used include anti-CD31 (phycoerythrinconjugated, Pharmingen), anti-CD41 (fluorescein isothiocynate [FITC] conjugated, Pharmingen), anti-CD54 (FITC conjugated, Pharmingen), antiCD 62E (FITC conjugated, Pharmingen), antiCD51 (FITC, Pharmingen), biotinylated-Ulex (Vector Laboratories), and anti-Annexin V (FITC conjugated, BD Biosciences). After this, 1ul of 0.2um filtered phosphate-buffered saline was added, allowing the sample to be ready for flow cytometry. For the Annexin $\mathrm{V}$ measurements, $5 \mathrm{mM}$ of $\mathrm{CaCl} 2$ was also present.

Because CD31 occurs on both eMPs and pMPs but CD41 occurs only on platelets, pMPs were defined as $C D 31^{+} \mathrm{CD} 41^{+}$events and eMPs as $C D 31^{+} \mathrm{CD} 41^{-}$ events. Events were counted by triggering on the red fluorescence signal of phycoerythrin, above background noise on the y-axis of the dot-plot, whereas the green signal of CD 41 was on the $\mathrm{x}$-axis 
(4-decade log scales, both $\mathrm{x}$ and $\mathrm{y}$ ). Fluorescence minus 1 control was used for accurate gating purposes. Microparticles were analyzed on a Coulter EPICs XL (Beckman Coulter, Miami, Florida) flow cytometer medium flow rate with a 30 -second stop time. The next task was to convert flow cytometer counts to the absolute value of eMPs per microliter of plasma. This was accomplished by using standard counting beads in which during a 30-second run time, $18 \mu \mathrm{L}$ of sample was actually aspirated on medium setting. Therefore, since 50 $\mu$ Lof PPP was utilized per run, a conversion factor calculated was $1178(\mathrm{~F}=[1.06 \mathrm{ml} / 0.018 \mathrm{ml}][1.0 \mathrm{ml} / 0.05 \mathrm{ml}])$.

Values are reported as counts/ $\mu \mathrm{L}$ of plasma. CD31 occurs also on some leukocyte subsets, but as previously observed using PE-Cy5-labeled CD45 4 leukocyte MPs contribute only a very small fraction of total CD $31^{+} \mathrm{CD} 41^{-}(<7 \%)$ in both controls and diseased participants and was therefore, neglected. To further validate this procedure, known amounts of pure pMPs and eMPs were prepared in vitro and mixed in various proportions to be then measured by this method; results were essentially the same as measuring the pure components individually. The eMPs 51,54, and 62E are not present in platelets in a detectable amount and were measured separately without co-incubation with CD41 antibody. Also, ULEX, CD 62P, and Annexin V were measured in a similar fashion. In each case, the detection of particles was set to trigger by fluorescence signal greater than noise. Fluorescent particles were further separated on another histogram by size by forward light scatter. Particles smaller than $1.5 \mu \mathrm{m}$ from the prior histogram were then analyzed on a third histogram, with those results recorded as the eMP count/ $\mu$ l. Due to the very high counts, to make more accurate measurements, ULEX samples were diluted 1:20. The resulting counts were multiplied by 20 to reflect the count/ $\mu \mathrm{L}$.

\section{Statistical Analysis}

A liberal strategy was employed to identify possible associations between MP levels and markers of COPD. Specifically, this meant attempting to elucidate a link between MP concentration levels and apoptotic ratio and any and all of the following: COPD status, GOLD stage (classification based on airflow limitation severity), ${ }^{18}$ DLCO, HRQoL questionnaire, BODE score, and acute exacerbations.
Control participants were assigned a GOLD stage of 0 for these purposes.

Unadjusted MP levels (and the apoptotic ratio, defined as eMP 62E divided by eMP 31) were considered, along with 2 adjusted levels. These adjusted levels reflected, for each participant, deviations from MP levels expected from conditions other than COPD, as determined by linear regression. One regression included as covariates age, gender, body mass index, smoking status, statin use, atherosclerosis or history of acute coronary syndrome, congestive heart failure, chronic kidney disease, connective tissue disease, dementia, diabetes with or without end organ damage, HIV infection, liver disease (mild, moderate, or severe), cancer with or without metastasis, peripheral artery disease, and peptic ulcer disease (Charlson score). The other regression included a pooled category of vascular disease and eliminated any covariates with fewer than 5 cases to dampen outlier effects.

Associations between adjusted and unadjusted MP levels and GOLD stage were tested for using standard analysis of variance, linear test of trend, and 2 contrast factors. This included comparing COPD patients to controls as well as COPD patients by stage compared to controls.

Associations between adjusted and unadjusted MP levels and the DLCO, SGRQ score, and the multidimensional BODE score were evaluated using the coefficient of determination $\left(\mathrm{R}^{2}\right)$.

All analyses were performed with NCSS 2004 (NCSS, LLC, Kaysville, Utah). Linear regression was performed on the subgroup of Stage 4 patients to correlate BODE score with MP levels.

\section{Results}

\section{Demographic Differences Between COPD Participants and Control Participants}

MP levels were measured in 19 control participants and 58 stable COPD participants. As expected, participants with COPD were older, had a higher pack-years smoking history, and lower lung function parameters. Not unsurprisingly, COPD participants also presented with more comorbidities than their controls (i.e., higher Charlson score) and had a higher percentage of statin and oxygen use (Table $1)$. 


\section{Table 1. Demographics and Clinical Characteristics of Both Control Participants and COPD Participants}

\begin{tabular}{|c|c|c|c|c|c|c|}
\hline \multirow[b]{2}{*}{ Parameters } & \multirow[b]{2}{*}{$\begin{array}{c}\text { Control } \\
\text { Participants }\end{array}$} & \multirow[b]{2}{*}{$\begin{array}{c}\text { COPD } \\
\text { Participants }\end{array}$} & \multicolumn{4}{|c|}{$\begin{array}{l}\text { COPD Participants by Classification } \\
\text { Based on Airflow Limitation Severity }\end{array}$} \\
\hline & & & 1 & 2 & 3 & 4 \\
\hline $\mathrm{N}$ & 19 & 58 & 8 & 18 & 19 & 13 \\
\hline Age (years) & $53 \pm 11$ & $65 \pm 11^{a}$ & $62 \pm 12$ & $62 \pm 13$ & $67 \pm 7$ & $68 \pm 8$ \\
\hline Male (\%) & $57.9 \%$ & $98.3 \%{ }^{\mathrm{b}}$ & $87.5 \%$ & $100 \%$ & $100 \%$ & $100 \%$ \\
\hline Ethnicity (W/AA/H) & $2 / 1 / 16$ & $33 / 12 / 13^{b}$ & $1 / 2 / 5$ & $9 / 2 / 7$ & $17 / 1 / 1$ & $6 / 7 / 0$ \\
\hline Active Smoker ('\%) & $36 \%$ & $31 \%{ }^{\mathrm{a}}$ & $37.5 \%$ & $38.8 \%$ & $36.8 \%$ & $7.6 \%$ \\
\hline Smoking (Pack Years) & $39 \pm 22$ & $65 \pm 52^{b}$ & $62 \pm 70$ & $52 \pm 38$ & $73 \pm 44$ & $74 \pm 54$ \\
\hline BODE Score & $\mathrm{N} / \mathrm{A}$ & $3 \pm 3^{b}$ & $0.75 \pm 1.2$ & $1.5 \pm 1.3$ & $4.1 \pm 2.3$ & $6.6 \pm 1.5$ \\
\hline Charlson Score & $0 \pm 1$ & $3 \pm 2^{\mathrm{b}}$ & $2.5 \pm 2.1$ & $3.3 \pm 1.7$ & $3.5 \pm 2.2$ & $4.2 \pm 2.9$ \\
\hline Statin Use (\%) & $15.8 \%$ & $43.1 \%^{\mathrm{b}}$ & $12 \%$ & $27.7 \%$ & $68 \%$ & $46 \%$ \\
\hline Oxygen Use (\%) & $0 \%$ & $27.6 \%{ }^{\mathrm{b}}$ & $12 \%$ & $5 \%$ & $26 \%$ & $69 \%$ \\
\hline \multicolumn{7}{|l|}{ Pulmonary Function } \\
\hline $\mathrm{FEV}_{1}(\mathrm{~L})$ & $3 \pm 0.6 \mathrm{~L}$ & $1.89 \pm 0.9 \mathrm{~L}$ & $3.42 \pm 0.7 \mathrm{~L}$ & $2.36 \pm 0.6 \mathrm{~L}$ & $1.46 \pm 0.2 \mathrm{~L}$ & $0.97 \pm 0.2 \mathrm{~L}$ \\
\hline Predicted FEV1(\%) & $94 \pm 11 \%$ & $52 \pm 23 \%$ & $93 \% \pm 6 \%$ & $66 \% \pm 12 \%$ & $42 \% \pm 5 \%$ & $28 \% \pm 6 \%$ \\
\hline $\mathrm{FEV}_{1} / \mathrm{FVC}$ & $96 \pm 10 \%$ & $56 \pm 15 \%$ & $71 \% \pm 13 \%$ & $67 \% \pm 12 \%$ & $48 \% \pm 8 \%$ & $42 \% \pm 8 \%$ \\
\hline $\mathrm{DLCO}(\mathrm{ml} / \mathrm{min} / \mathrm{mmHg})$ & $27.6 \pm 5.3$ & $16.6 \pm 7.1$ & $22.5 \pm 4.9$ & $21 \pm 7.6$ & $13.4 \pm 3.7$ & $11.4 \pm 3.5$ \\
\hline Predicted DLCO (\%) & $101 \pm 21 \%$ & $59 \pm 21 \%$ & $80 \% \pm 12 \%$ & $72 \% \pm 18 \%$ & $50 \% \pm 13 \%$ & $42 \% \pm 14 \%$ \\
\hline
\end{tabular}

${ }_{\mathrm{a}}^{\mathrm{a}} \mathrm{p}>0.05$

$\mathrm{N}=$ number of participants in each cohort

Age, smoking pack years, Charlson score, pulmonary function all presented as mean + /-standard deviation.

$\mathrm{DLCO}=$ diffusing capacity of the lungs for carbon monoxide; W/AA/H=White/African American/Hispanic; $\mathrm{FEV}_{1}=$ forced expiratory volume in 1 second; FVC=forced vital capacity; $\mathrm{COPD}=$ chronic obstructive pulmonary disease; $\mathrm{BODE}=\mathrm{Body}$ mass index-airflow ObstructionDyspnea-Exercise index; GOLD=Global initiative for chronic Obstructive Lung Disease

\section{Microparticle Levels in Control Participants versus COPD Participants}

The unadjusted values in control participants versus COPD participants show similar medians for all MP levels and the apoptotic ratio. All comparisons were not statistically significant even when adjusted for age, gender, BMI, smoking status, statin use, and comorbidities (Figure 1).

\section{Microparticle Levels in Control Participants versus COPD Participants by GOLD Stage}

MP levels in control participants versus COPD participants by GOLD stage show similar medians for most MP levels (Figure 2). However, there was a statistically significant increase in ULEX and pMP $62 \mathrm{P}^{+}$among COPD stage 3 participants compared to controls ( $p=0.01$, Figures $2 \mathrm{a}$ and $2 c$ ).

Interestingly when we compared the apoptosis level by eMP $62 \mathrm{E}^{+} / \mathrm{eMP} 31^{+}$ratio we noticed that there was a statistically significant $(p<0.05)$ increase in apoptotic ratio among stage 2 and stage 3 COPD participants compared independently to either controls or stage 1 COPD participants (Figure 2e). This suggests that non-COPD and less severe COPD individuals may actually exhibit increased apoptosis compared to more severe COPD individuals.

\section{Microparticle Level Correlation with BODE, DLCO and Quality of Life Scores}

The association between MPs and BODE score, DLCO, and quality of life scores was evaluated by linear regression adjusting for important variables known to alter MP levels. We found no correlation between MPs and the percent predicted DLCO (Figure 3). We were also unable to find a significant correlation between MPs and quality of life scores 


\section{Figure 1. Levels of Endothelial and Platelet Microparticles in Control Participants Versus COPD Participants}
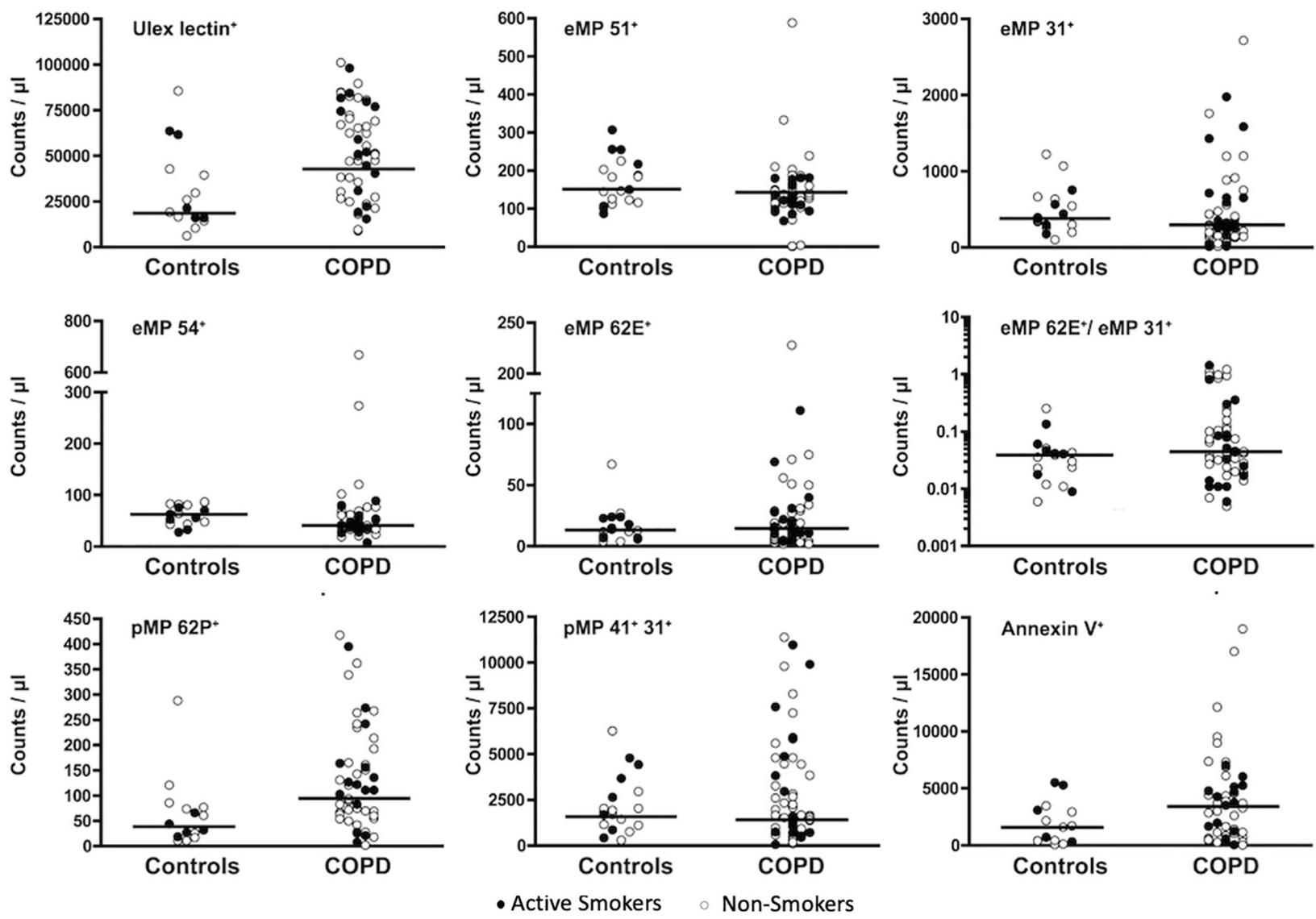

All comparisons (Figure 1a-1i) were not statistically significant $(p>0.05)$. The levels of eMPs (ULEX lectin $\left.{ }^{+}, \mathrm{CD} 31^{+}, \mathrm{CD}^{2} 1^{+}, \mathrm{CD}^{2} 4^{+}, \mathrm{CD} 62 \mathrm{E}^{+}\right) \mathrm{pMPs}$ $\left(\mathrm{CD} 41^{+} 31^{+}, \mathrm{CD} 62 \mathrm{P}^{+}\right)$and apoptotic MPs (Annexin $\mathrm{V}^{+}, \mathrm{CD} 2 \mathrm{E}^{+} / \mathrm{CD} 31^{+}$ratio) were not statistically different between controls and COPD participants

eMPs=endothelial microparticles; $\mathrm{COPD}=$ chronic obstructive pulmonary disease; $\mathrm{pMPs}=$ platelet microparticles

(Figure 4). Finally, when we looked at that association between BODE score and MPs (Figure 5), the coefficient of determination $\left(R^{2}\right)$ for the association of pMP $41^{+} 31^{+}$and the BODE score showed a mild, yet statistically significant correlation $\mathrm{R}^{2}=0.019$ $(p=0.004)$ (Figure 5f).

\section{Microparticle Levels in Acute Exacerbation Versus Stable COPD Participants}

In 17 patients we obtained MP levels in both acute exacerbations and in the stable state (Figure 6). There was no difference in the apoptotic ratio as well as most MPs levels when paired comparisons were made. The only MP that had a statistically significant difference in concentration (decreased during acute exacerbation) was ULEX lectin $(P=0.04)$. (Figure 6a)

\section{Microparticle Levels in Stage 4 Participants}

In 9 patients with COPD GOLD Stage 4, eMPs and pMPs were correlated with severity by BODE score (Figure 7). There is a strong association between BODE score and the pMP levels. BODE score explains $75 \%$ of the variability seen in pMP $41^{+} 31^{+}$ levels $\left(\mathrm{R}^{2}=0.75\right)$. (Figure $\left.7 \mathrm{a}\right)$.

\section{Discussion}

Our study centered around measuring various endothelial and platelet-derived microparticles in relation to relevant COPD features and exacerbation status. This hypothesis was based on the literature that shows COPD is associated with increased endothelial apoptosis and dysfunction as well as 


\section{Figure 2. Levels of Endothelial and Platelet Microparticles in Control Participants Versus COPD Participants Divided by GOLD Stage}

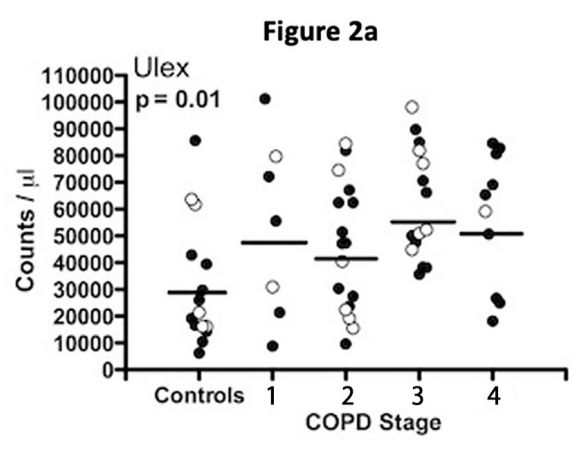

Figure 2d

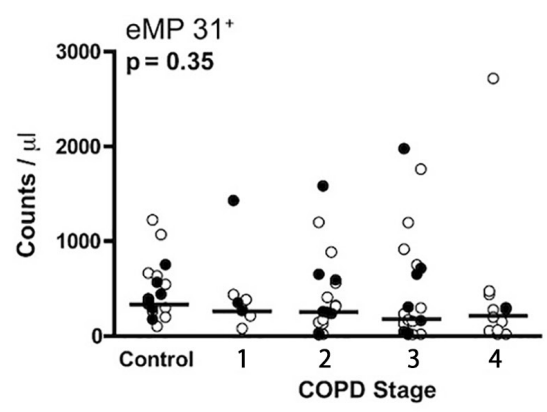

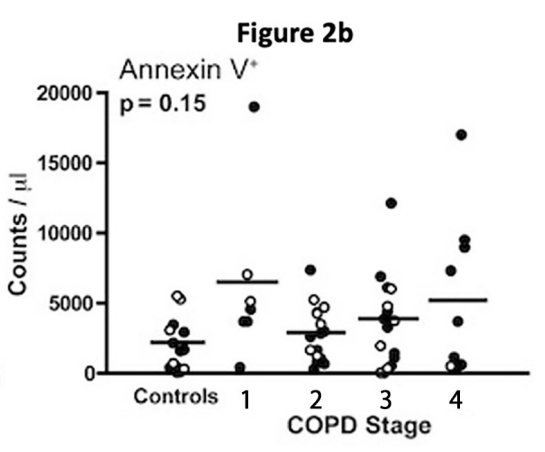

Figure 2e

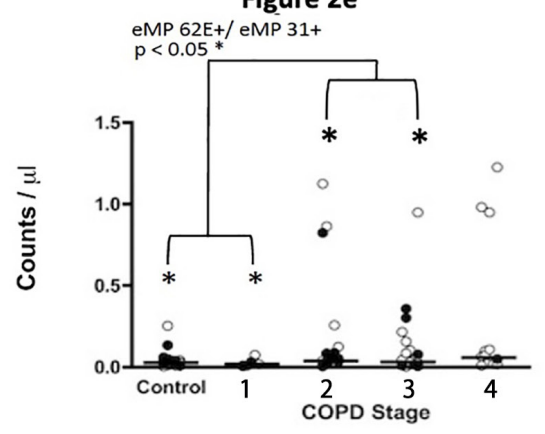

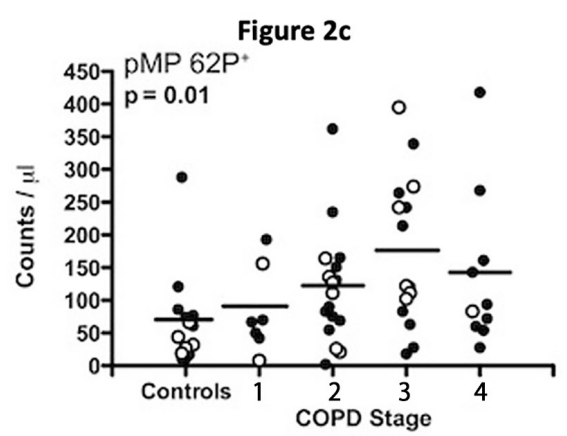

Figure $\mathbf{2 f}$

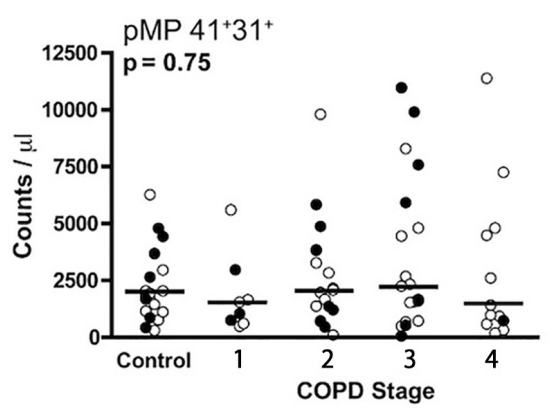

- Active Smokers

- Non-Smokers

ULEX (Figure 2a) and pMP $62 \mathrm{P}^{+}($Figure $2 \mathrm{c}$ ) were increased among COPD stage 3 patients compared to controls $(p=0.01)$. The apoptotic ratio eMP $62 \mathrm{E}^{+} / 31^{+}$(Figure $2 \mathrm{e}$ ) was increased among stage 2 and 3 COPD patients compared independently to either controls or stage 1 COPD patients $(p<0.05)$ Levels of Annexin $\mathrm{V}^{+}$, eMP $31^{+}$, pMP $41^{+} 31^{+}$(Figures $2 \mathrm{~b}, 2 \mathrm{~d}, 2 \mathrm{f}$ ) as well as eMP $62^{+}$, eMP $54^{+}$, eMP51+ (not shown) were not statistically significantly different $(p>0.05)$.

$\mathrm{COPD}=$ chronic obstructive pulmonary disease; $\mathrm{eMPs}=$ endothelial microparticles; pMPs=platelet microparticles

platelet dysfunction/hypercoagulability. We did not find any difference in the level of MPs between control participants and COPD participants. These results were still apparent even after adjusting for confounding factors like smoking history, age, coronary artery disease, etc.

Previous studies have reported that COPD individuals have increased $\mathrm{CD} 31^{+}$(PECAM) and MPs compared to controls as well as to less severe COPD individuals. ${ }^{27,28}$ Takahashi et al $(\mathrm{N}=127)$ reported this same association with $\mathrm{CD} \mathrm{E}^{+}$( $\mathrm{E}$ selectin), however, the larger Multi-Ethnic Study of Atherosclerosis COPD study (MESA) $(\mathrm{N}=180)$ was unable to show a statistically significant difference between $\mathrm{CD} 6 \mathrm{E}^{+}$or $\mathrm{CD} 51^{+}$MP's between COPD individuals and controls. ${ }^{27}$ Lastly, Takahashi et al reported that both $\mathrm{CD}_{3} 1^{+}$and $\mathrm{CD} 62 \mathrm{E}^{+} \mathrm{MP}$ were elevated during acute exacerbations but we were unable to show this same conclusion. These discrepancies may be explained by population differences and variations in sample recollection and processing. Our study used a validated methodology processed in a laboratory with ample experience in these types of measures. $4,12,29$

ULEX is an MP of particular interest although few studies have incorporated its use in characterizing eMPs in COPD, possibly due to reports that the lectin ULEX is an unsuitable biomarker for endothelium for in vivo studies due to its lack of specificity. ${ }^{6}$ Nevertheless, previous publications support its tropism for human endothelium. ${ }^{30,31}$ Furthermore, mechanistic studies into the pathophysiology of COPD have used ULEX lectincoated magnetic beads to isolate human pulmonary endothelium $^{32}$ suggesting that it may in fact be useful for examining changes in COPD-related lung endothelium. Interestingly, $\mathrm{ULEX}^{+}$lectin $^{+}$ was the only MP elevated in COPD stage 3 patients 


\section{Figure 3. Association Between Microparticles and Percent Predicted Diffusing Capacity of the Lung for Carbon Monoxide}

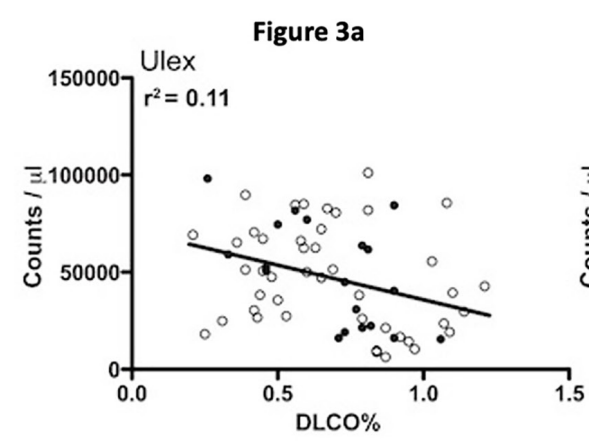

Figure 3d

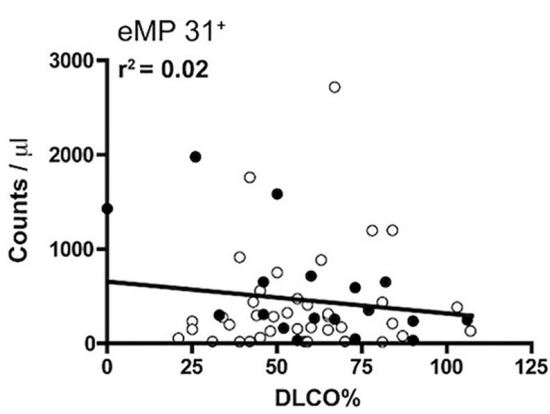

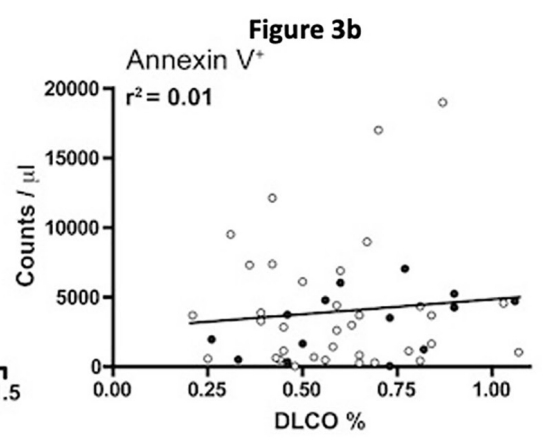

Figure 3e

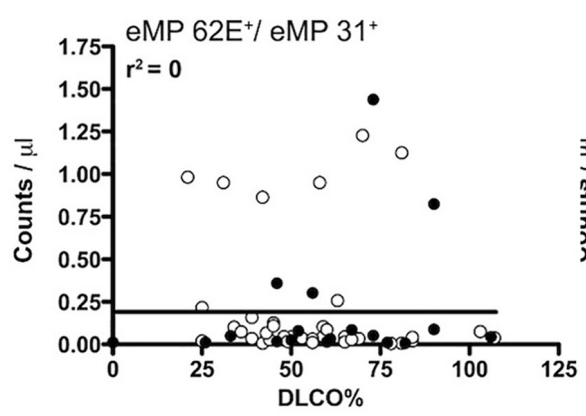

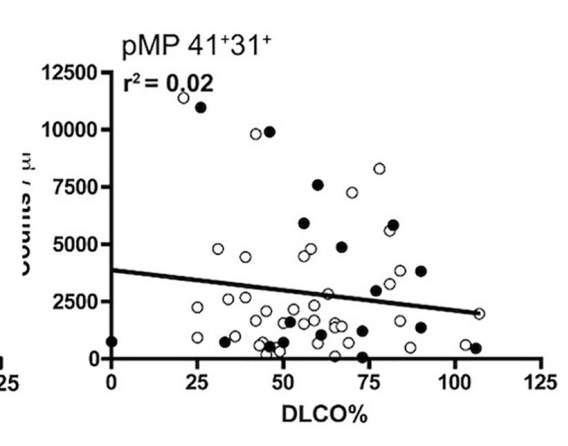

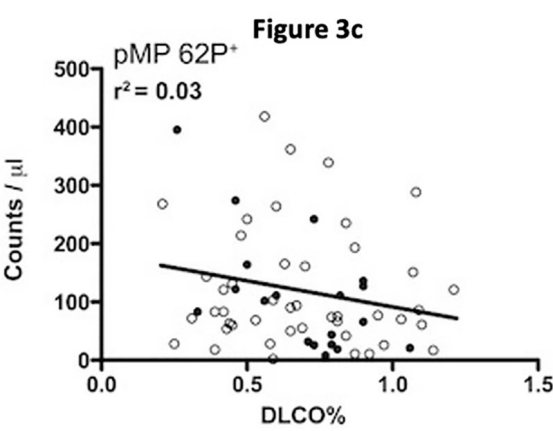

Figure $3 f$

- Active Smokers $\circ$ Non-Smokers

There was no statistically significant association between percent predicted DLCO and all microparticles studied including eMP $62 \mathrm{E}^{+}$, eMP $54^{+}$, eMP $51^{+}$ (not shown).

$\mathrm{DLCO}=$ diffusing capacity of the lung for carbon monoxide; pMPs=platelet microparticles eMPs=endothelial microparticles;

compared to controls (Figure 2, $p=0.01$ ). We also clearly detected that ULEX lectin ${ }^{+}$MP levels drop during acute COPD exacerbations with later clinical stabilization (Figure $6 a, p=0.04$ ). Given that certain microRNAs upregulated in COPD patients can inhibit proper endothelial tube formation and sprouting, ${ }^{32}$ it is possible that more advanced COPD-induced endothelial dysregulation results in less $\mathrm{ULEX}^{+}$microparticles/epitopes being expressed in pulmonary vasculature and thus, an increase in COPD individuals' plasma. During COPD exacerbations, acute-onset repair mechanisms may explain decreased ULEX ${ }^{+}$lectin measured in patient plasma at this stage. Further research in this area could help recognize $\mathrm{ULEX}^{+}$as a potential marker of COPD exacerbations.

We also did not find a clear association between MPs and either DLCO or HRQOL which matches previous studies. For example, the MESA group showed that while CD $31^{+}$eMP levels were inversely associated with DLCO, in their fully adjusted model, there was no association between either $C D 51^{+}$or $\mathrm{CD} 6 \mathrm{E}^{+}$with diffusing capacity. ${ }^{27}$ This highlights 2 things: the inherent heterogeneity when it comes to measuring MPs as well as the fact that certain ones may be better indicators than others of structural lung dysfunction.

One unique analysis of our study was to assess the eMP $62 \mathrm{E}^{+} / \mathrm{eMP} 31^{+}$apoptotic ratio in this population. A lower ratio means higher levels of endothelial apoptosis as mentioned before. We found that early-stage smokers (controls and stage 1 COPD) independently had increased apoptosis compared with more advanced COPD stages. These findings are in line with a previous report showing that smokers with normal spirometry and low DLCO have a lower eMP $62 \mathrm{E}^{+} / \mathrm{eMP} 31^{+}$ratio in comparison with participants with normal spirometry, normal DLCO, nonsmokers. ${ }^{33}$ This is likely a reflection of ongoing endothelium damage at early stages 


\title{
Figure 4. Association Between Microparticles and Quality of Life Scores
}
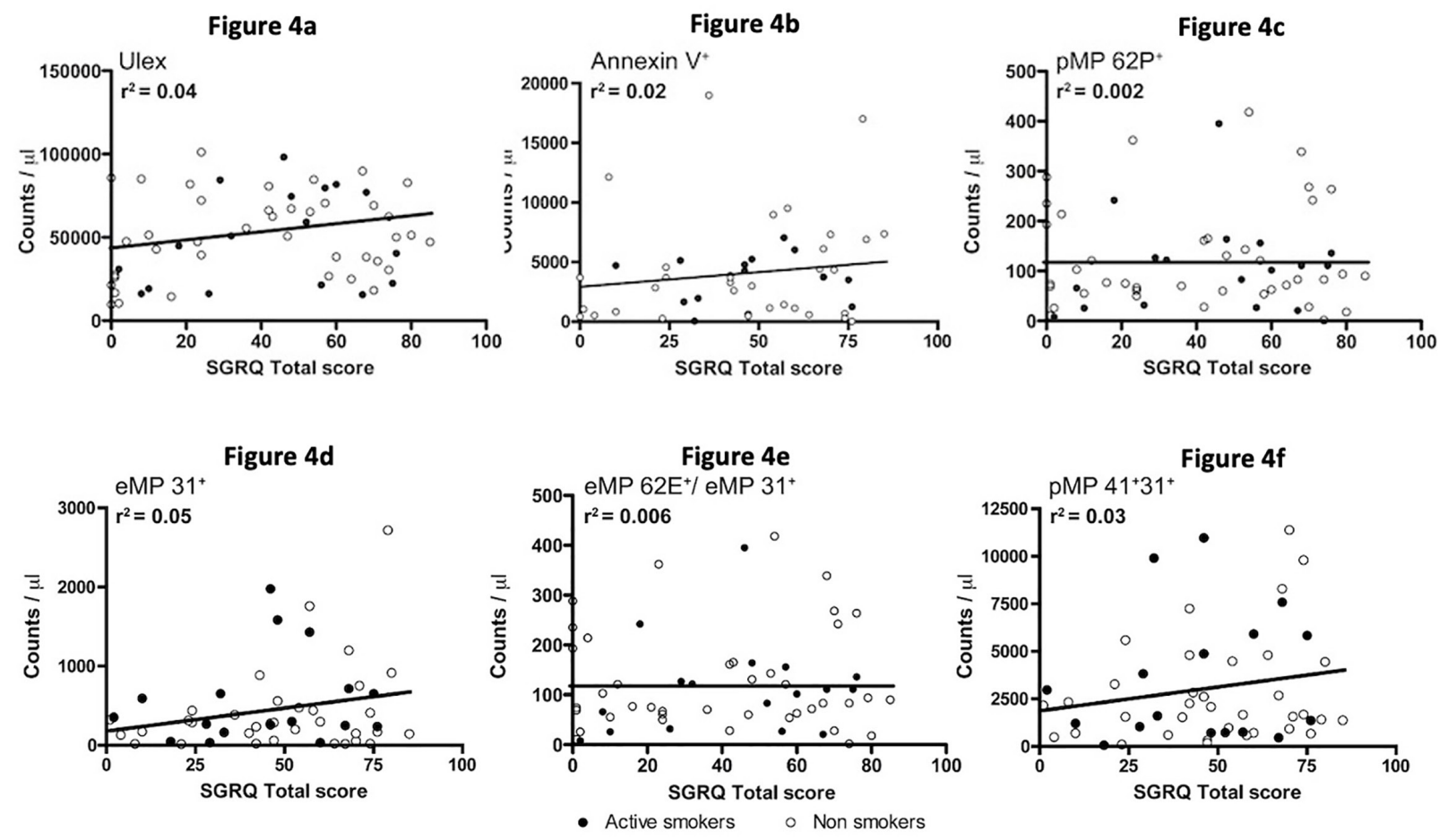

- Active Smokers

Non-Smokers

\begin{abstract}
There was no statistically significant association between quality-of-life scores and all microparticles studied including eMP $62 \mathrm{E}^{+}, \mathrm{eMP} 54^{+}$, eMP $51^{+}$ (not shown).

St George's Respiratory Questionnaire=SGRQ; eMPs=endothelial microparticles; pMPs=platelet microparticles
\end{abstract}

of the disease when most participants do not have clinical evidence of disease. Better understanding of the apoptotic ratio might help early recognition of COPD as well as early interventions to prevent progression of the disease.

Furthermore, the MESA COPD Study ${ }^{27}$ found that $\mathrm{CD} 31^{+}$eMPs were elevated in both mild COPD and participants with emphysema compared to controls while $\mathrm{CD}^{2} \mathrm{E}^{+}$eMPs, indicative of endothelial activation, were elevated only in severe COPD participants and participants with hyperinflation. While our group was unable to show this isolated increase in $C D 62 \mathrm{E}^{+}$eMPs among severe COPD participants, likely due to our smaller sample size, our results highlight that the use of the apoptotic ratio is a marker sensitive enough to reflect the apoptosis and activation process that occurs in COPD that should be further explored.

MP assessment in COPD may be of increased value in assessing the pathophysiologic processes that occur in late COPD. We found a minor, yet statistically significant association between pMP $41^{+} / 31^{+}$and the BODE score (Figure 6, $p=0.004$, $\left.\mathrm{R}^{2}=0.019\right)$ overall, but this association became stronger and clearer when we look at our COPD stage 4 participants (Figure $7, p=0.002, \mathrm{R}^{2}=0.75$ ). This may suggest increased platelet activation as the disease progresses. Several publications have demonstrated the association between thrombocytosis and platelets activation with increased 1-year mortality after COPD exacerbation and a protective role of antiplatelet therapy in patients with acute exacerbations. $^{20,34}$ Our findings are intriguing because to our knowledge, the association between increased pMPs with BODE score within a single cohort of stage 4 COPD patients has never been described before, making a new potential connection between worsening outcomes and platelets activation. 


\section{Figure 5. Association Between Microparticles and BODE Score}

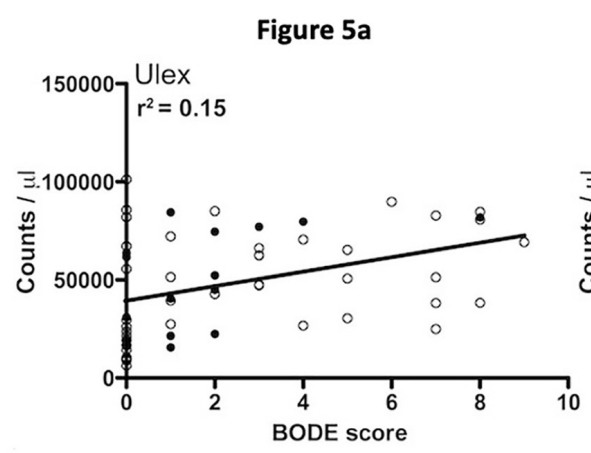

Figure 5d

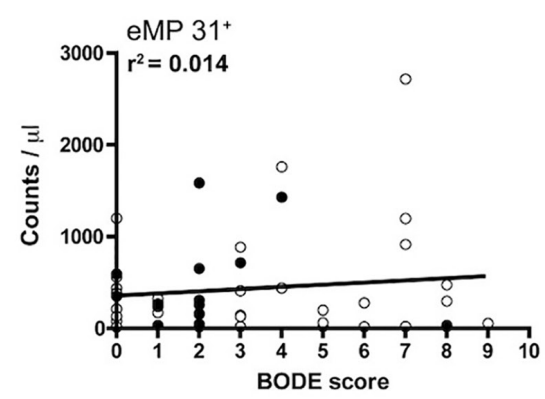

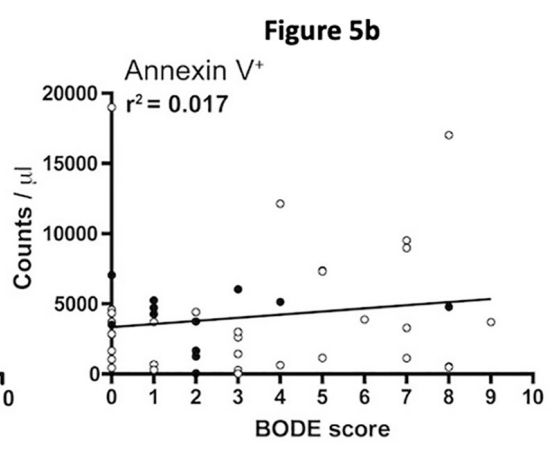

Figure $5 \mathbf{f}$
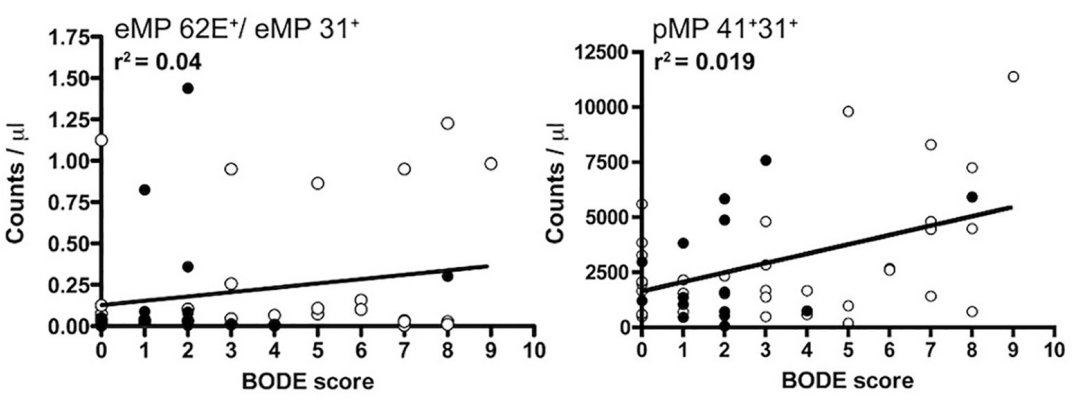

- Active Smokers

- Non-Smokers

There is a mild, yet statistically significant correlation between pMP $41^{+} 31^{+}$and BODE score $\left(\mathrm{R}^{2}=0.019\right)$ (Figure $\left.5 f, p=0.004\right)$. There was no statistically significant difference for all other microparticles studied including eMP $62 \mathrm{E}^{+}$, eMP $54^{+}$, eMP $51^{+}$(not shown).

BODE=Body mass index-airflow Obstruction-Dyspnea-Exercise index; eMPs=endothelial microparticles; pMPs=platelet microparticles

\section{Limitations}

Some limitations of our study may explain some of our discrepant results compared to similar studies. The most important is probably sample collection and processing techniques. MP levels are not normally distributed and show great variability between patients. Furthermore, the inherent process of collecting serum and plasma specimens, preparing them for flow cytometry, and measuring MP levels offer several opportunities for variability. ${ }^{35,36}$ For example, it has been reported that simple agitation may further increase MP levels in serum samples ${ }^{35}$ and MP measurement techniques lack standardization, thus contributing to differences in recorded values. ${ }^{36,37}$ We collected our samples following a strict protocol and samples were processed in a laboratory with years of expertise in performing these techniques. ${ }^{5}$ The other important limitation is sample size, as our study involved a fewer number of participants compared to others, ${ }^{27,28}$ thus a lack of power may explain the negative results. Nevertheless, our study has a size comparable to other studies, ${ }^{32,38,39}$ and has highlighted how analysis of some MPs (such as the apoptotic ratio) can be readily assessed in smaller populations. Lastly, a significant portion of our study population was on statin therapy which, due to its anti-inflammatory role, may impact the formation, release, and number of MPs. Further studies are needed to quantify this impact.

\section{Conclusion}

We found that most MPs measured do not correlate significantly with COPD status, COPD severity, or exacerbations in our cohort. The apoptotic eMP $62 \mathrm{E}^{+} / \mathrm{eMP} 31^{+}$ratio may be a useful marker of early endothelium apoptosis and early recognition of the disease process. Lastly, platelet activation assessed 


\section{Figure 6. Paired Comparisons of Endothelial and Platelet Microparticle Levels of Participants at Stable State Versus Acute Exacerbation}

Figure 6a

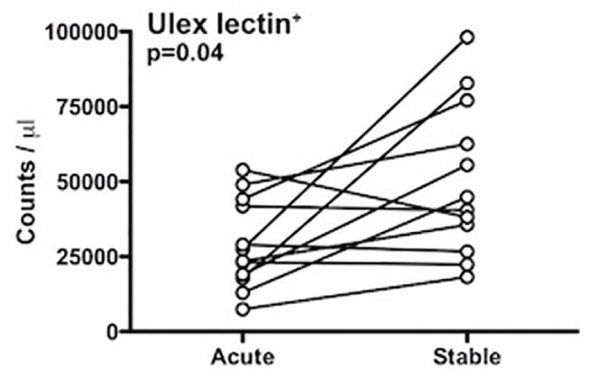

Figure 6d

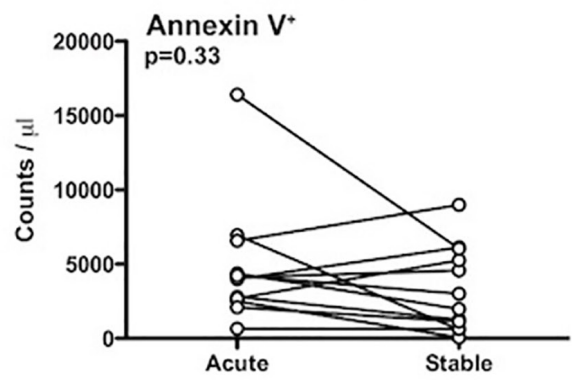

Figure $6 \mathrm{~g}$

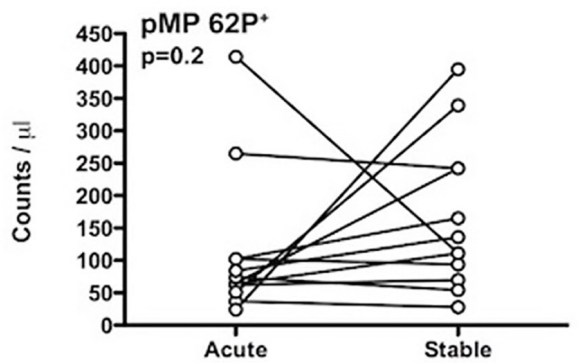

Figure 6b

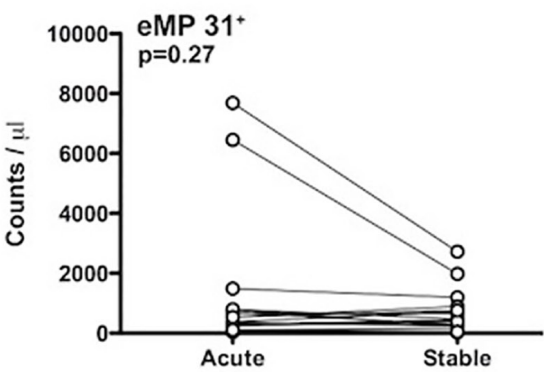

Figure 6e

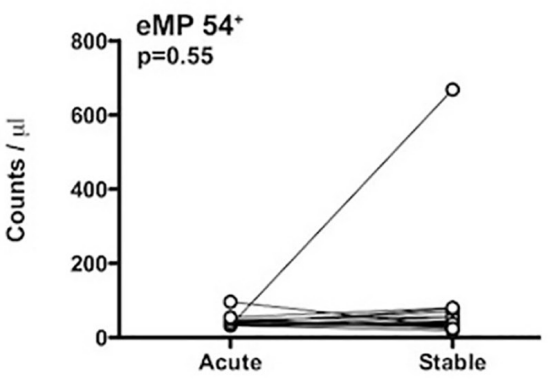

Figure $6 \mathrm{~h}$

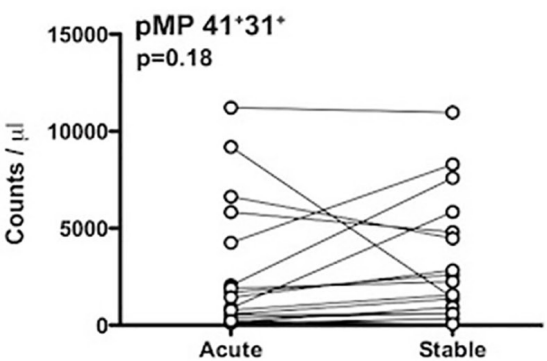

Figure 6c

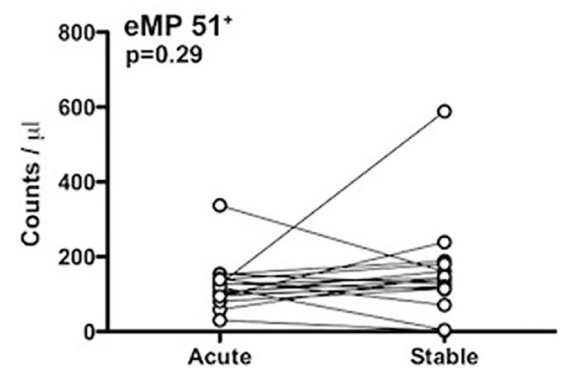

Figure $6 f$

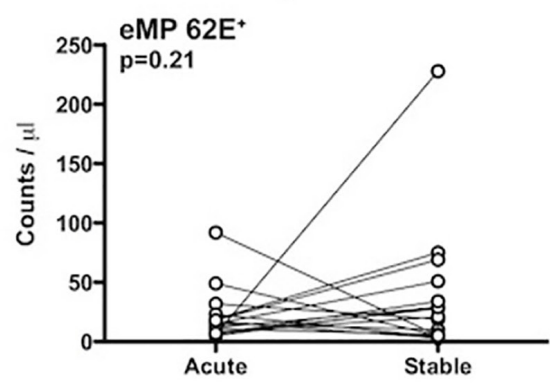

Figure $6 \mathbf{i}$

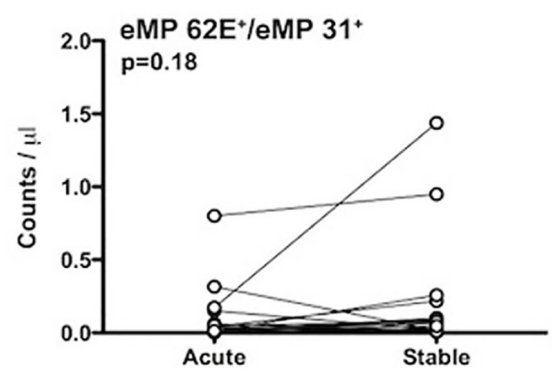

There was no statistically significant difference between stable condition and exacerbation except for ULEX lectin+ $($ Figure $6 a, p=0.04)$.

eMPs=endothelial microparticles; pMPs=platelet microparticles

by $\mathrm{pMP} 41^{+} 31^{+}$increases with disease severity and may be an important feature for stage 4 COPD patients.

\section{Acknowledgments}

Author contributions: JK was responsible for organizing, writing, and drafting the first draft of the manuscript as well as formatting figures, tables and statistical analyses. JL was responsible for coordinating the participant recruitment, performing clinical examinations, data collection/ organization, and final editing of the manuscript.
MC was responsible for study design, clinical examinations, participant recruitment, coordinating specimen laboratory measurement, and final editing of the manuscript. MC collected patient samples/ data as well as statistical analyses, figure creation, and initial drafting of the manuscript. All authors have contributed to the intellectual content of the article and gave approval for publication.

\section{Declaration of Interest}

The authors report no conflicts of interest in this work. 


\section{Figure 7. Association Between Microparticles and BODE Score in Stage 4 COPD Patients}

Figure 7a

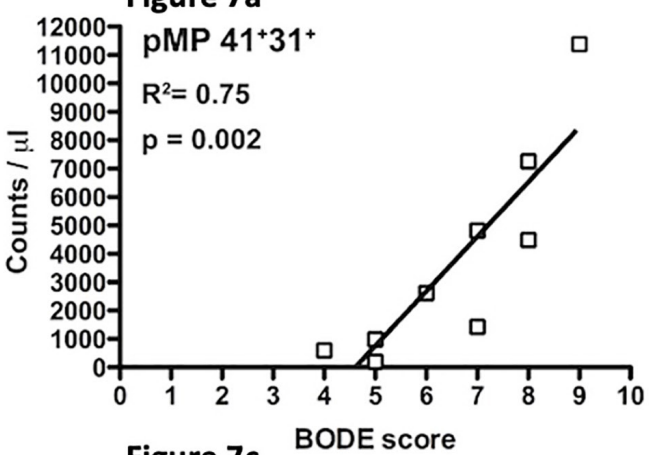

Figure 7c

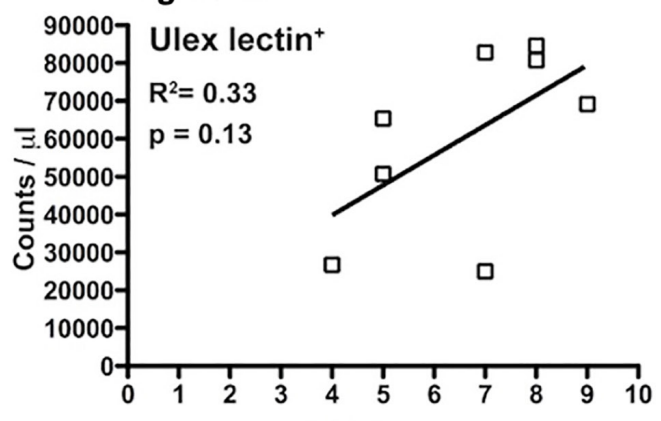

Figure 7e BODE score

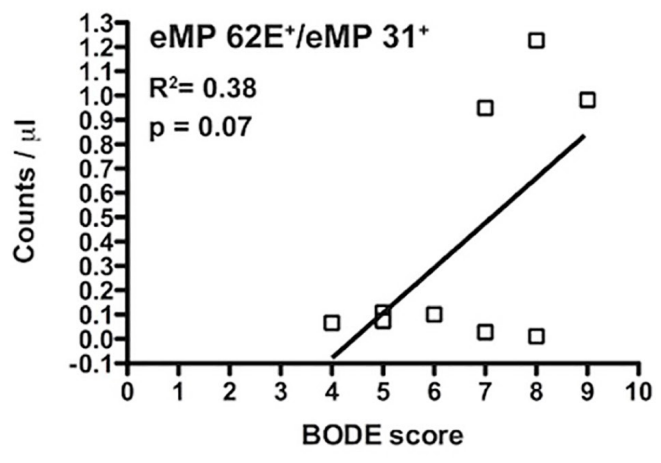

Figure 7b

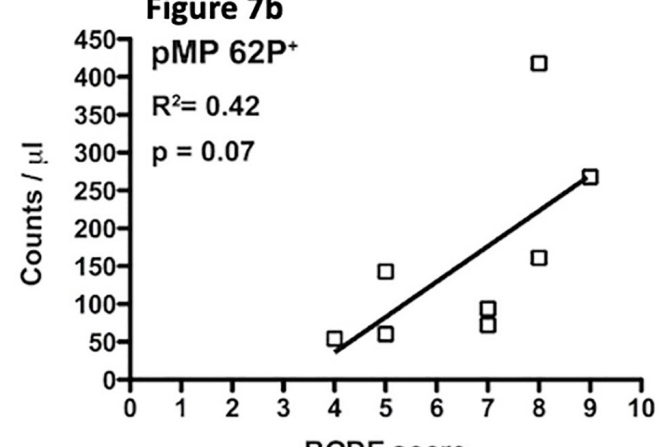

Figure 7d

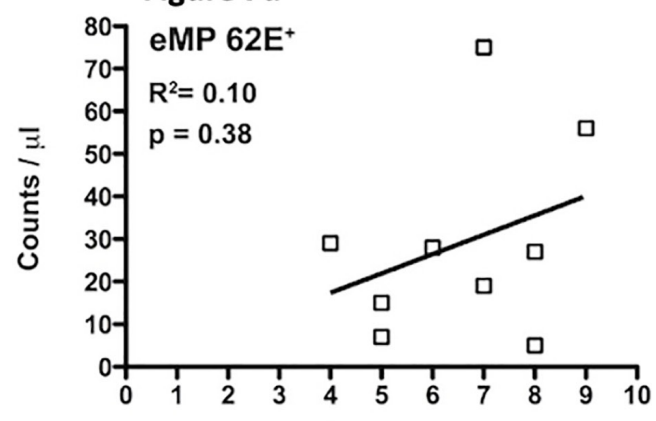

Figure 7f BODE score

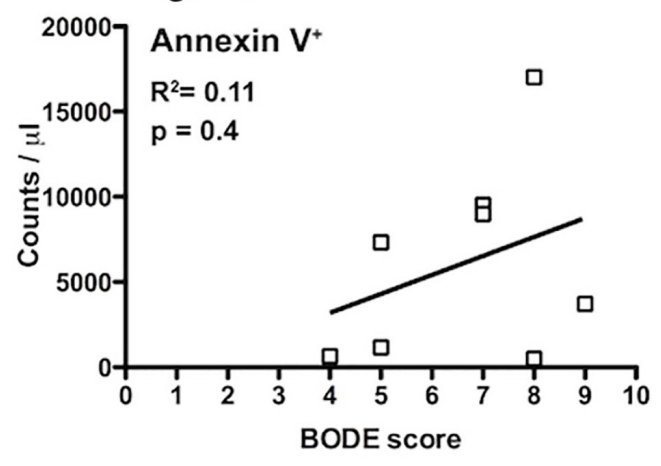

There is a strong association between pMP41+31+ levels and BODE score (Figure $7 a, p=0.002$ ).

BODE=Body mass index-airflow Obstruction-Dyspnea-Exercise index; eMPs=endothelial microparticles; pMPs=platelet microparticles 


\section{References}

1. Chironi GN, Boulanger CM, Simon A, Dignat-George F, Freyssinet JM, Tedgui A. Endothelial microparticles in diseases. Cell Tissue Res. 2009;335(1):143-151.

doi: https://doi.org/10.1007/s00441-008-0710-9

2. Mackman N. On the trail of microparticles. Circ Res. 2009;104(8):925927. doi: https://doi.org/10.1161/CIRCRESAHA.109.196840

3. Distler JH, Huber LC, Gay S, Distler O, Pisetsky DS. Microparticles as mediators of cellular cross-talk in inflammatory disease. Autoimmunity. 2006;39(8):683-690.

doi: https://doi.org/10.1080/08916930601061538

4. Horstman LL, Ahn YS. Platelet microparticles: a wide-angle perspective. Crit Rev Oncol Hematol. 1999;30(2):111-142. doi: https://doi.org/10.1016/S1040-8428(98)00044-4

5. Soriano AO, Jy W, Chirinos JA, et al. Levels of endothelial and platelet microparticles and their interactions with leukocytes negatively correlate with organ dysfunction and predict mortality in severe sepsis. Crit Care Med. 2005;33(11):2540-2546.

doi: https://doi.org/10.1097/01.CCM.0000186414.86162.03

6. Horstman LL, Jy W, Jimenez JJ, Ahn YS. Endothelial microparticles as markers of endothelial dysfunction. Front Biosci. 2004;9(2):11181135. doi: https://doi.org/10.2741/1270

7. Hristov M, Erl W, Linder S, Weber PC. Apoptotic bodies from endothelial cells enhance the number and initiate the differentiation of human endothelial progenitor cells in vitro. Blood. 2004;104(9):27612766. doi: https://doi.org/10.1182/blood-2003-10-3614

8. Distler JHW, Huber LC, Hueber AJ, et al. The release of microparticles by apoptotic cells and their effects on macrophages. Apoptosis. 2005;10(4):731-741.

doi: https://doi.org/10.1007/s10495-005-2941-5

9. Jimenez JJ, Jy W, Mauro LM, Soderland C, Horstman LL, Ahn YS. Endothelial cells release phenotypically and quantitatively distinct microparticles in activation and apoptosis. Thromb Res. 2003;109(4):175-180.

doi: https://doi.org/10.1016/S0049-3848(03)00064-1

10. Combes V, Simon AC, Grau GE, et al. In vitro generation of endothelial microparticles and possible prothrombotic activity in patients with lupus anticoagulant. J Clin Invest. 1999;104(1):93-102.

doi: https://doi.org/10.1172/JCI4985

11. Owens AP, Mackman N, Weber C, Mause S. Microparticles in hemostasis and thrombosis. Circ Res. 2011;108(10):1284-1297. doi: https://doi.org/10.1161/CIRCRESAHA.110.233056

12. Arteaga RB, Chirinos JA, Soriano AO, et al. Endothelial microparticles and platelet and leukocyte activation in patients with the metabolic syndrome. Am J Cardiol. 2006;98(1):70-74. doi: https://doi.org/10.1016/j.amjcard.2006.01.054
13. Amabile N, Guignabert C, Montani D, Yeghiazarians Y, Boulanger CM, Humbert M. Cellular microparticles in the pathogenesis of pulmonary hypertension. Eur Respir J. 2013;42(1):272-279. doi: https://doi.org/10.1183/09031936.00087212

14. Bernal-Mizrachi L, Jy W, Jimenez JJ, et al. High levels of circulating endothelial microparticles in patients with acute coronary syndromes. Am Heart J. 2003;145(6):962-970. doi: https://doi.org/10.1016/S0002-8703(03)00103-0

15. Amabile N, Guerin AP, Leroyer A, et al. Circulating endothelial microparticles are associated with vascular dysfunction in patients with end-stage renal failure. J Am Soc Nephrol. 2005;16(11):33813388. doi: https://doi.org/10.1681/ASN.2005050535

16. Bakouboula B, Morel O, Faure A, et al. Procoagulant membrane microparticles correlate with the severity of pulmonary arterial hypertension. Am J Respir Crit Care Med. 2008;177(5):536-543. doi: https://doi.org/10.1164/rccm.200706-8400C

17. Chambers E, Rounds S, Lu Q. Pulmonary endothelial cell apoptosis in emphysema and acute lung injury. In: Parthasarathi K, eds. Molecular and Functional Insights into the Pulmonary Vasculature. Advances in Anatomy, Embryology and Cell Biology. Springer International Publishing;2017:63-86. doi: https://doi.org/10.1007/978-3-319-68483-3_4

18. Global Initiative for Chronic Obstructive Lung Disease (GOLD). Global strategy for the diagnosis, management and prevention of chronic obstructive pulmonary disease. 2018 report. GOLD website. Published 2018. Accessed January 2021. https://goldcopd.org/ wp-content/uploads/2017/11/GOLD-2018-v6.0-FINAL-revised-20Nov_WMS.pdf

19. Skoczyński S, Krzyżak D, Studnicka A, et al. Chronic obstructive pulmonary disease and platelet count. In: Pokorski M, ed. Advances in Pulmonary Medicine: Research and Innovations. Springer International Publishing; 2019:19-23. doi: https://doi.org/10.1007/5584_2019_379

20. Harrison MT, Short P, Williamson PA, Singanayagam A, Chalmers JD, Schembri S. Thrombocytosis is associated with increased shortand long-term mortality after exacerbation of chronic obstructive pulmonary disease: a role for antiplatelet therapy? Thorax. 2014;69(7):609-615.

doi: https://doi.org/10.1136/thoraxjnl-2013-203996

21. Rabe KF, Hurst JR, Suissa S. Cardiovascular disease and COPD: dangerous liaisons? Eur Respir Rev. 2018;27(149):180057. doi: https://doi.org/10.1183/16000617.0057-2018

22. Ekström MP, Hermansson AB, Ström KE. Effects of cardiovascular drugs on mortality in severe chronic obstructive pulmonary disease. Am J Respir Crit Care Med. 2013; 187(7):715-720. doi: https://doi.org/10.1164/rccm.201208-1565OC 
23. Rabe KF, Hurd S, Anzueto A, et al. Global strategy for the diagnosis, management, and prevention of chronic obstructive pulmonary disease: GOLD executive summary. Am J Respir Crit Care Med. 2007;176(6):532-555.

doi: https://doi.org/10.1164/rccm.200703-456SO

24. Jones PW, Quirk FH, Baveystock CM, Littlejohns P. A self-complete measure of health status for chronic airflow limitation: the St. George's respiratory questionnaire. Am Rev Respir Dis. 1992;145(6):13211327. doi: https://doi.org/10.1164/ajrccm/145.6.1321

25. Donaldson GC, SeemungalTA, BhowmikA, WedzichaJA. Relationship between exacerbation frequency and lung function decline in chronic obstructive pulmonary disease. Thorax. 2002;57(10):847-852. doi: https://doi.org/10.1136/thorax.57.10.847

26. Jimenez JJ, Jy W, Mauro LM, Horstman LL, Ahn YS. Elevated endothelial microparticles in thrombotic thrombocytopenic purpura: findings from brain and renal microvascular cell culture and patients with active disease. Br J Haematol. 2001;112(1):81-90. doi: https://doi.org/10.1046/j.1365-2141.2001.02516.x

27. Thomashow MA, Shimbo D, ParikhMA, etal. Endothelialmicroparticles in mild chronic obstructive pulmonary disease and emphysema. the multi-ethnic study of atherosclerosis chronic obstructive pulmonary disease study. Am J Respir Crit Care Med. 2013;188(1):60-68. doi: https://doi.org/10.1164/rccm.201209-16970C

28. Takahashi T, Kobayashi S, Fujino N, et al. Increased circulating endothelial microparticles in COPD patients: a potential biomarker for COPD exacerbation susceptibility. Thorax. 2012;67(12):1067-1074. doi: https://doi.org/10.1136/thoraxjnl-2011-201395

29. Jy W, Horstman L, Ahn Y. Microparticle size and its relation to composition, functional activity, and clinical significance. Semin Thromb Hemost. 2010;36(08):876-880. doi: https://doi.org/10.1055/s-0030-1267041

30. Miettinen M, Holthofer H, Lehto V-P, Miettinen A, Virtanen I. Ulex europaeus I lectin as a marker for tumors derived from endothelial cells. Am J Clin Pathol. 1983;79(1):32-36.

doi: https://doi.org/10.1093/ajcp/79.1.32

31. Hormia M, Lehto V, Virtanen I. Identification of UEA I-binding surface glycoproteins of cultured human endothelial cells. Cell Biol Int Rep. 1983;7(6):467-475.

doi: https://doi.org/10.1016/0309-1651(83)90136-4

32. Green CE, Bicknell R, Turner AM. S113 Defining the molecular signature of the pulmonary endothelium in chronic obstructive pulmonary disease (COPD). Thorax. 2017;72(Suppl 3):A69. doi: https://doi.org/10.1136/thoraxjnl-2017-210983.119

33. Gordon C, Gudi K, Krause A, et al. Circulating endothelial microparticles as a measure of early lung destruction in cigarette smokers. Am J Respir Crit Care Med. 2011;184(2):224-232. doi: https://doi.org/10.1164/rccm.201012-2061OC
34. Maclay JD, McAllister DA, Johnston S, et al. Increased platelet activation in patients with stable and acute exacerbation of COPD. Thorax. 2011;66(9):769-774.

doi: https://doi.org/10.1136/thx.2010.157529

35. Gyorgy B, Szabo TG, Pasztoi M, et al. Membrane vesicles, current state-of-the-art: emerging role of extracellular vesicles. Cell Mol Life Sci. 2011;68(16):2667-2688.

doi: https://doi.org/10.1007/s00018-011-0689-3

36. Ayers L, Kohler M, Harrison P, et al. Measurement of circulating cellderived microparticles by flow cytometry: sources of variability within the assay. Thromb Res. 2011;127(4):370-377. doi: https://doi.org/10.1016/j.thromres.2010.12.014

37. Puddu P, Puddu GM, Cravero E, Muscari S, Muscari A. The involvement of circulating microparticles in inflammation, coagulation and cardiovascular diseases. Can J Cardiol. 2010;26(4):140-145. doi: https://doi.org/10.1016/S0828-282X(10)70371-8

38. Mobarrez F, Antoniewicz L, Bosson JA, Kuhl J, Pisetsky DS, Lundbäck M. The effects of smoking on levels of endothelial progenitor cells and microparticles in the blood of healthy volunteers. PLoS One. 2014;9(2):e90314.

doi: https://doi.org/10.1371/journal.pone.0090314

39. Tőkés-Füzesi M, Ruzsics I, Rideg O, Kustán P, Kovács GL, Molnár T. Role of microparticles derived from monocytes, endothelial cells and platelets in the exacerbation of COPD. Int JChron Obstruct Pulmon Dis. 2018;13:3749-3757.

doi: https://doi.org/10.2147/COPD.S175607 